Archives de sciences sociales des religions

184 | octobre-décembre 2018

Bulletin bibliographique

\title{
Vincent DELECROIX, Apocalypse du politique
}

Paris, Desclée de Brouwer, 2016, 363 p.

\section{Benjamin Fabre}

\section{OpenEdition}

Journals

Édition électronique

URL : https://journals.openedition.org/assr/44777

DOI : $10.4000 /$ assr.44777

ISSN : $1777-5825$

Éditeur

Éditions de l'EHESS

Édition imprimée

Date de publication : 1 décembre 2018

Pagination : 264-266

ISSN : 0335-5985

\section{Référence électronique}

Benjamin Fabre, «Vincent DELECRoIx, Apocalypse du politique », Archives de sciences sociales des religions [En ligne], 184 | octobre-décembre 2018, mis en ligne le 01 décembre 2018, consulté le 10 janvier 2022. URL : http://journals.openedition.org/assr/44777 ; DOI : https://doi.org/10.4000/assr. 44777

Ce document a été généré automatiquement le 10 janvier 2022.

(c) Archives de sciences sociales des religions 


\section{Vincent DELECROIX, Apocalypse du politique}

Paris, Desclée de Brouwer, 2016, 363 p.

\section{Benjamin Fabre}

\section{RÉFÉRENCE}

Vincent DELECRoIX, Apocalypse du politique, Paris, Desclée de Brouwer, 2016, 363 p.

1 Le beau mot d'«apocalypse " est à prendre ici dans toute la richesse de ses significations. Porté par le texte de l'évangéliste Jean, ce mot désigne d'abord la révélation : quelque chose qui était caché se montre désormais. Ce dévoilement est en même temps un bouleversement, vite éprouvé comme un cataclysme. La chose qui se montre ne fait pas qu'apparaître, elle ouvre tout un champ où elle-même peut sembler disparaitre. Ainsi en est-il de la politique, longtemps cachée, invisible sous des masques divers, elle se révèle parfois brutalement, ne laissant pas intact le champ où elle se jouait. Vraie catastrophe, s'ouvre quelque chose de méconnaissable qui désoriente et déconcerte la société et peut tout aussi bien être mortel que salvifique. Vincent Delecroix laisse ouvert l'avenir du politique, plus optimiste que beaucoup.

2 L'auteur part de Rome pour déterminer clairement la nouveauté de l'avatar chrétien du théologico-politique. Mais le théologico-politique a une bien plus vieille histoire. Avant que les notions de théologie, de politique, ou même celle de Dieu ou de polis soient présentes, les groupes humains n'ont pas pu se limiter à une vision horizontale de leurs relations internes. Pierre Clastres ou Cornelius Castoriadis ont bien montré, avec d'autres, que la société a toujours eu besoin d'un ancrage vertical, qui fonde en quelque sorte son unité et lui confère une sorte de substantialité. L'ordre cosmique semble le garant de l'ordre social, des dieux aux chefs une hiérarchie et une analogie s'établissent, qu'il s'agisse d'une fusion, d'une délégation, d'une mission: les formes en ont été multiples. Si l'auteur avait élargi sa vision à ces formes archaïques du théologico-politique, il aurait sans doute mieux encore fondé la difficulté considérable 
que nous avons à en penser de nouvelles, et surtout celles instaurées par le christianisme. L'auteur se limite à un champ européen : sur les affaires du ciel et de la terre, le Fils du Ciel aurait aussi son mot à dire.

Quoi qu'il en soit, c'est ici le christianisme qui vient bouleverser leur rapport politique. Car le Royaume du Ciel n'est pas un règne terrestre. Force est donc à celui-ci de se penser à une certaine distance, dans une certaine tension, avec le lieu spirituel où les hommes ne font pas la loi. Les lois humaines peuvent être inspirées par Dieu, mais elles ont des problèmes à régler qui ne sont pas ceux de l'éternité. Dieu et le Roi peuvent être complices, si l'on peut dire, mais Dieu est plus roi que le Roi et le Roi n'est pas Dieu. Ce qui se passe, sous les ordres du Roi terrestre, ne peut qu'être aboli, plutôt que réalisé, dans le royaume divin. Et comme toute distance, celle-ci peut engendrer contradiction ou même divorce : il y a des tyrans dont l'ordre est diabolique. Et le soutien divin que ceux-ci peuvent invoquer devient alors une imposture où se disloque leur dialogue.

Quand le pouvoir politique ne peut plus "se dédoubler pour s'affirmer", comment pourra-t-il se légitimer? La métaphore fragilisée cherche à se maintenir sans la croyance qui la soutenait. Elle ne disparait pas, et l'auteur nous invite à suivre cette métaphore perdue.

Métaphore perdue dont le fantôme hante nos démocraties. Dans la deuxième partie de son ouvrage, consacré au passé du théologico-politique, l'auteur revient souvent sur les perspectives déjà tracées dans sa longue analyse de la notion même. Une logique interne semble mener le théologico-politique vers ce qu'on a appelé sa sécularisation, d'un terme ambigu qui pourrait aussi bien viser sa dissolution que sa traduction évolution étudiée attentivement par l'auteur dans Ce n'est point ici le pays de la vérité (voir ASSR, 176). Il y a dans le christianisme une possibilité de "déconstruire la souveraineté », nous dit-il ici. L'institution politique doit refouler en permanence le rappel de son illégitimité ou tout au moins de son caractère contingent. Les guerres de religion ont contribué à rendre douteux les liens du ciel et de la terre.

Dans son Cours de philosophie positive, à la 54 ${ }^{\text {ème }}$ Leçon, Auguste Comte notait: "Cette longue et déplorable phase de la désintégration finale du catholicisme [...] a été systématisée par la grande institution de la célèbre Compagnie de Jésus qui, de nature éminemment rétrograde, fut alors spécialement fondée, avec un admirable instinct politique, pour servir d'organe central à la résistance générale du catholicisme contre la destruction universelle dont il était directement menacé par l'essor croissant de l'émancipation spirituelle » (p. 311). On n'est plus aussi sûr que la religion ait tant à perdre dans cette perte. Et beaucoup ont même trouvé là plutôt une chance de renouveau qu'une fin de partie. Il est bien vrai que la « résistance » signalée par Comte s'est aussi manifestée dans ce que l'auteur appelle "sinistre désir du religieux ». Anxieux devant son effacement et ranimé par les incertitudes démocratiques, un recours déboussolé au religieux cherche parfois à retrouver l'ancienne harmonie. Comme l'a bien souvent dit Friedrich Nietzsche, la mort de Dieu n'est pas seulement une bonne nouvelle. L'auteur montre bien que le lieu même où se fait sentir la perte de la transcendance, c'est la question de la souveraineté. Si le pouvoir politique ne participe plus de l'autorité attribuée à Dieu, souverain du cosmos, son fondement est à chercher dans l'horizontalité fonctionnelle des rapports sociaux. C'est « le triomphe du droit sur le souverain", nous dit l'auteur, mais le droit suppose un consensus problématique. 
7 Je voudrais souligner l'intérêt de l'analyse que fait l'auteur de la dissolution de la notion de souveraineté : ce n'est pas seulement le Prince qui a cessé d'être divin, c'est Dieu qui a cessé d'être royal. Le christianisme a tenté de substituer à l'image du Dieu couronné au sein de sa Cour angélique un Christ souffrant, porteur de toute la condition humaine. Si le Christ roi continue de hanter certains milieux, le Christ crucifié peut difficilement être l'image d'un pouvoir politique puissant.

L'auteur ne nous laisse pas devant ce spectacle d'une société aux valeurs flottantes, ballotée entre scepticisme nihiliste et fanatisme archaïsant. L'intérêt peut-être le plus grand de son livre est de tenter un retour de la transcendance qui ne serait pas la résurrection d'un passé caduque. Il consacre à cet effort la dernière partie de son travail qu'il intitule «Hantise du royaume ». L'auteur aboutit donc à ce constat : «La mise en cause du Dieu de l'onto-théologie, de l'attribut de la puissance souveraine, de l'image du Roi des rois ou du souverain de l'Être [...], cette déconstruction suscite un autre rapport de la philosophie à la parole biblique» (p. 260). Il faut surmonter la tentation d'insérer le Royaume dans un futur historique. Attendre de l'Histoire qu'elle aboutisse au Royaume restaurerait ce que l'auteur appelle le «joug religieux ». Il faut « détacher le messianisme de la croyance au progrès ». La pensée du Royaume doit être une " hantise ", et non pas une prédiction. Ce qui hante, c'est ce qui à la fois est là et ne peut y être. Le Royaume devrait devenir la «conscience critique», la "mauvaise conscience» de la société. Il ne s'agit plus du réconfort de la vision d'une société providentielle, mais de l'inquiétude et du refus qui naissent de celle d'une société injuste, injuste depuis toujours, et dont rien ne permet d'attendre une régénération quelconque. La pensée du Royaume devient celle de l'impossible - à partir de quoi il faut pourtant espérer que tout n'est pas dit et que le monde si atroce soit-il n'est pas condamné. Qu'est-ce alors que l'espérance ? C'est le tourment « du rien ontologique qui manque à la communauté réelle». Voilà bien l'Apocalypse du politique : le politique apparaît dans la nudité de rapports sociaux toujours marqués par l'oppression et la mystification et ce dévoilement pourrait bien être la catastrophe où rien de positif n'adviendra jamais. À moins... à moins de croire que quelque chose se joue dans cette confuse mêlée que nous appelons l'histoire, mais sur quoi étayer une telle confiance si elle n'est pas un optimisme béat? Peut-on imaginer une communauté cimentée malgré tout par une croyance sans objet? Le "Principe Espérance " d'Ernst Bloch peut-il donner à une société le lien qu'elle ne peut plus tirer d'un passé démystifié ? En évoquant ici « l'image désarticulée » du théologico-politique, l'auteur espère sans doute rétablir un rapport, fût-il mystérieux, entre la transcendance et le misérable ici-bas. Le globe Terraqué, comme l'appelait Ronsard, échappe à la noyade... mais le monde où nous vivons nous permet-il de compter sur des citoyens de cette trempe? « Le fond de tout, disait en 1940 un curé de la Drôme à André Malraux, c'est qu'il n'y pas de grande personne... ». Ces grandes personnes devraient-elle être croyantes pour porter en elles cette nouvelle figure du théologico-politique? Devraient-elles entretenir l'ombre d'un mythe qui serait l'unique obstacle à une re-mythologisation néo-païenne ? Comme l'a écrit Bloch, «seul un chrétien peut faire un bon athée ». Le chrétien aurait-il conservé cette tension qui arrache l'espérance aux dérisoires perspectives des triomphes terrestres? Un appel, une aspiration, un élan qui «brisent le temps » par la promesse messianique ? Avec confiance, l'auteur évoque en terminant cette mission de la religion qui ne rétablirait pas le fantasme théocratique, mais «ouvre incontestablement à la politique jamais réalisable ». 
9 «Incontestablement », dit l'auteur. Cet adverbe et les belles analyses qui le soutiennent peuvent laisser le lecteur pour le moins perplexe. On peut en effet se poser incontestablement? - deux questions : jusqu'au siècle dernier, c'est bien une utopie qui semblait se substituer au rêve paradisiaque. Si confuse fût-elle, elle dessinait pourtant les contours vagues d'un autre temps, d'un temps de la réconciliation, d'une sortie de la préhistoire dramatique de l'humanité. C'était à tout le moins un support imaginaire qui rassemblait des efforts trop souvent désespérants. Si l'on constate aujourd'hui que ce fantôme d'un futur encore trop naïvement religieux se dissipe absolument, peut-on penser un élan des masses humaines devenu assez mûr pour se passer de toute imagerie ? L'unité sociale peut-elle reposer alors sur la communauté d'une volonté de justice et de fraternité universellement partagée? Autre question: les sociétés contemporaines aspirées dans les tourbillons de la mondialisation nous approchentelles de cet espoir ? À l'heure où des responsables politiques nous disent tout de go que la société n'existe pas, qu'il n'y a que des individus qui ont à se débrouiller dans une jungle où ils sont responsables de leur destin, faut-il s'étonner que nos concitoyens soient tiraillés entre l'individualisme le plus étroit et la tentation d'un retour à des temps plus heureux? Les élucubrations sophistiquées et stupides de la Silicon Valley vont-elles devenir la simiesque figure de la Parousie?

Il faut beaucoup d'optimisme - peut-être donc de foi - pour espérer voir prendre forme ce visage libéré et transformé du théologico-politique. S'il ne s'agit pas seulement de rénover un agencement conceptuel mis à mal par les temps que nous vivons, mais de guetter les signes d'une nouvelle vision du politique dans la réalité concrète des pratiques citoyennes, l'horizon peut sembler bien incertain. Entre ceux qui méprisent, ceux qui oublient, et ceux qui corrompent le politique, le chemin est étroit pour ceux qui pensent encore que quelque chose d'essentiel y soit en jeu. 